\title{
Enhancing Traditional Recommender Systems via Social Communities
}

\author{
Leschek Homann, Denis Mayr Lima Martins, \\ Gottfried Vossen* and Karsten Kraume \\ European Research Center \\ for Information Systems (ERCIS) \\ University of Münster, Leonardo-Campus 3, \\ 48149 Münster, Germany \\ *vossen@wi.uni-muenster.de
}

Received 17 November 2018

Accepted 17 November 2018

Published 14 January 2019

\begin{abstract}
Collaborative Filtering (CF) has become the most popular approach for developing Recommender Systems in diverse business applications. Unfortunately, problems such as the cold-start problem (i.e., new users or items enter the system and for those no previous preference information is available) and the gray sheep problem (i.e., cases in which a user profile does not match any other profile in the user community) are widely recognized for hindering recommendation effectiveness of traditional CF methods. To alleviate such problems, substantial research has focused on enhancing $\mathrm{CF}$ with social information about users (e.g., social relationships and communities). However, despite the crescent interest in social-based approaches, researches and practitioners face the challenge of developing their own Recommender System architecture for appropriately combining social and collaborative filtering methods to improve recommendation results. In this paper, we address this issue by introducing a flexible architecture to support researchers and practitioners in the task of designing real-world Recommender Systems that exploit social network data. We focus on detailing our proposed architecture modules and their interplay, potential algorithms for extracting and combining relevant social information, and candidate technologies for handling diverse and massive data volumes. Additionally, we provide an empirical analysis demonstrating the effectiveness of the proposed architecture on alleviating the cold-start problem over a concrete experimental case.
\end{abstract}

Keywords: Recommender Systems; social communities; cold-start problem.

\section{Introduction}

Recommender Systems (RS) have been widely adopted in substantially diverse business scenarios, varying from movie and song recommendations to next-best

This is an Open Access article published by World Scientific Publishing Company. It is distributed under the terms of the Creative Commons Attribution 4.0 (CC-BY) License. Further distribution of this work is permitted, provided the original work is properly cited. 
action suggestions in the industrial sector. The massive and crescent volume of information being generated everyday has motivated the development of such systems with the primary focus on delivering relevant items (e.g., products, news, professional connections, job vacancies, etc.) according to user needs and preferences. In this context, Collaborative Filtering (CF) methods have become the most popular approach producing recommendations, since such methods majorly exploit on preference patterns (e.g., ratings) in the user community in order to deliver unseen, relevant items to users, rather than relying on highly unstructured and complex content-specific information about items in a catalog. However, the advantage of traditional CF methods also comes with a drawback $^{1}$ : such methods are widely recognized to suffer from problems such as (a) cold-start (i.e., new users or recently added items for those no previous preference information is available) and (b) gray sheep (i.e., cases in which a user profile does not match any other profile in the user community). To alleviate these problems, researchers have proposed the use of external data describing user social behavior (e.g., social relationships and communities) so that more elaborate preference patterns can be exploited by $\mathrm{CF}^{2}$; this is also the topic of the present paper.

$\mathrm{CF}$ produces recommendations by estimating or predicting user ratings for unrated items based on existing ratings given by other users. ${ }^{1}$ In this sense, items with the highest estimated ratings are likely to be of interest to the user and, therefore, the system presents these items to her. More specifically, a Recommender System tries to estimate the relevance of an item $i$ from an itemset $I$ for a user $u$ from a set $U$ of users using a prediction method. Such a prediction method exploits patterns in previous ratings $r_{u, i}, u \in U=\left\{u_{l}: l=1,2, \ldots, n_{u}\right\}$ and $i \in I=\left\{i_{k}: k=1,2, \ldots, n_{w}\right\}$, where $n_{u}$ is the number of users and $n_{w}$ is the number of items in the system, respectively. Since no historical data about new users is available, it is not possible to find users with alike interests, which leads $\mathrm{CF}$ to produce inappropriate recommendations.

The use of social network data to enhance CF-based Recommender Systems and alleviate the aforementioned problems has shown promising results. ${ }^{2}$ Such social-based recommenders exploit user relations stored in a social graph where nodes represent users and edges depict different relationship types among users (e.g., family bonds, friendship, etc.). For instance, the tie strength between users can be considered to produce more accurate recommendations, particularly in cases where a spare rating dataset hinders the effectiveness of $\mathrm{CF}^{3}$ In a previous work, ${ }^{4}$ we have introduced a conceptual framework that describes how social collective signals can be incorporated in traditional Recommender Systems to alleviate the cold-start problem. Next, we present an illustrative example to clarify the concept of social-based recommendations.

Running example. Assume that video streaming company VideoComp is interested in attracting new users to its platform. The company decides to reach users from a social-media website by showing videos in their timeline, as Fig. 1 shows. To make the action more effective, targeted users (i.e., Nico and Jan) should be connected to VideoComp's clients (i.e., Julia, Felix, and Jens) in social media, and suggested videos should be relevant to the new users. By employing our framework, 
Clients

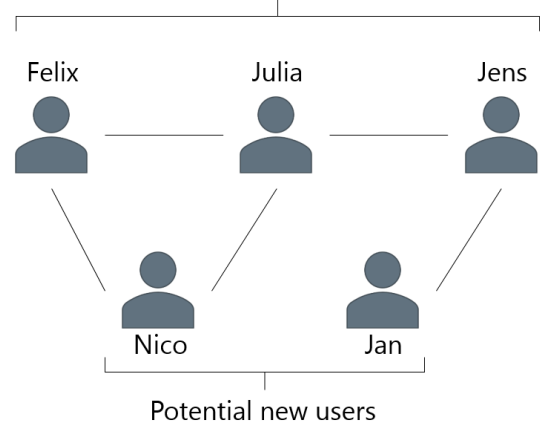

$\left.\begin{array}{|c|c|c|}\multicolumn{1}{l}{\text { User }} & \multicolumn{1}{c}{\text { Video A }} & \text { Video B } \\ \hline \text { Julia } & \text { Like } & \text { Like } \\ \hline \text { Felix } & \text { Like } & \text { Dislike } \\ \hline \text { Jens } & \text { Dislike } & \text { Like } \\ \hline \text { Nico } & \text { Like } & - \\ \hdashline \text { Jan } & - & \text { Like }\end{array}\right]$ - $\begin{gathered}\text { Predicted } \\ \text { ratings }\end{gathered}$

Fig. 1. Running example. A video recommendation on social media.

the company can exploit clients' data (i.e., video ratings) to recommend relevant videos to new users. In this case, Video A would be recommended to Nico, while Video B would appear in Jan's timeline.

In this paper, we extend the proposed framework by presenting a detailed implementation and experimental analysis that demonstrates its effectiveness. The contribution of this paper is twofold, as we provide a general architecture and a first approach on how to measure the accuracy of its provided recommendations.

The rest of this paper is organized as follows: Section 2 presents the foundations of our framework. Section 3 describes a general recommender architecture and a concrete implementation. Section 4 reports results obtained via an experimental evaluation considering different performance metrics. Section 5 presents a brief discussion about the limitations of this work. Section 6 offers an overview of related researches. Section 7 concludes this paper with an outline of future work.

\section{Social Collective Signals for Recommender Systems}

The social collective framework proposed in Homann et al. ${ }^{4}$ was designed to exploit relations and patterns in a user community in order to provide useful recommendations to users that are new to a recommendation platform. To achieve this goal we apply a community detection algorithm on social network data to identify groups of users (i.e., communities) that appropriately capture different user preferences. Whenever recommendations are required to a new user, we find to which group this target user belongs and use existing ratings given by users in his or her group to generate recommendations (e.g., by applying a popularity-based recommendation algorithm). This approach is particularly interesting since the size of the often used user-item matrix is smaller for each group of users, which reduces the processing time required to generate recommendations.

An overview of this social collective framework is depicted in Fig. 2. The Community Detection and Management module - marked as I in Fig. 2 - is 


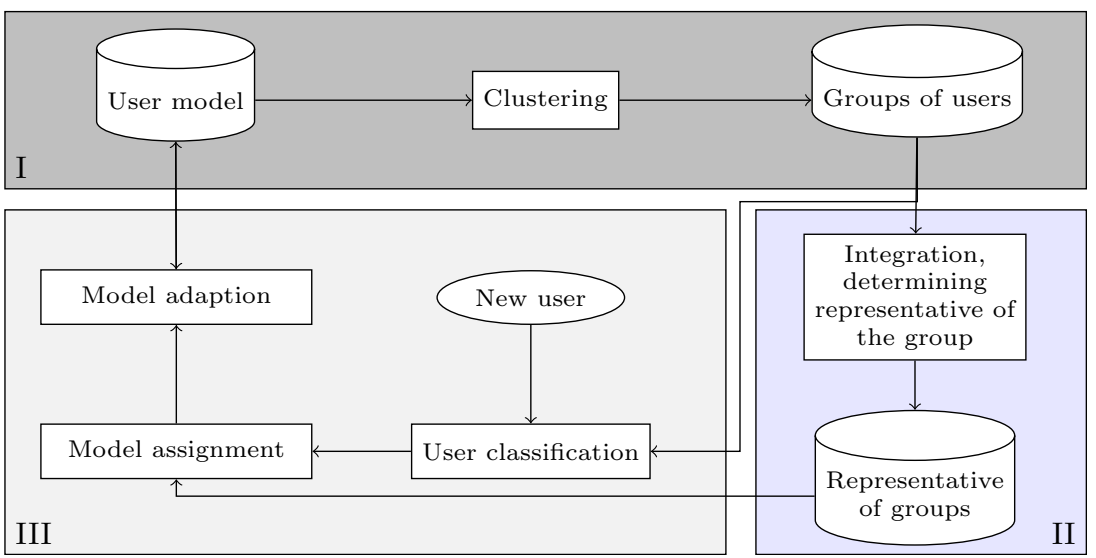

Source: Extracted from Ref. 4.

Fig. 2. Conceptual schema of the proposed generic framework.

responsible to process social network data ${ }^{\mathrm{a}}$ in order to find user communities and store them in a database. User communities are identified by community detection algorithms such as betweenness-oriented or density-oriented ones. ${ }^{5}$ This module is also responsible for regularly updating the communities based on changes in the user preferences or in the social network (e.g., new user connections in the social graph).

The Representative module (Fig. 2, II) is responsible to determine recommendations based on ratings given by users within a community. Such recommendations may be determined by a popularity-based or an aggregation-based algorithm such as calculating average community ratings and recommending the highest-rated items.

Finally, the Assignment and Adaptation module (Fig. 2, III) is responsible for finding a community to which a new user belongs and present the recommendations produced by the Representative module. The activities of the new user are observed and the provided recommendations are adapted according to new information about the user (e.g., active feedback).

Next, we describe the general architecture that allows $\mathrm{CF}$ enhancements via social-based signals.

\section{Architecture and Implementation}

\subsection{General architecture}

In this subsection, we describe the general architecture of a collaborative Recommender System and how it can be enhanced to take advantage of social information about users. Figure 3 shows a general architecture and the components it comprises.

a A social graph in which nodes correspond to users and edges represent user-user relationships such as friendship or following. 


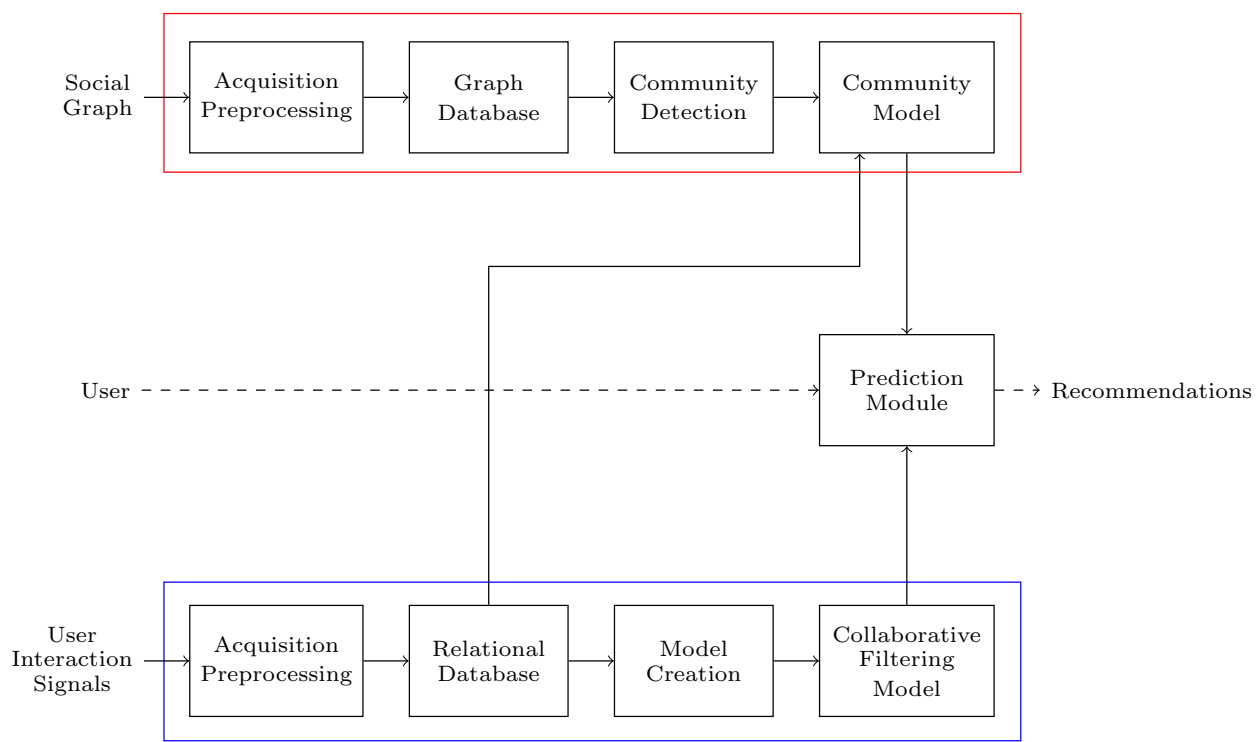

Fig. 3. General architecture of a Recommender System with social enhancement to alleviate the coldstart problem.

The architecture is divided into two parts. In the lower part of the architecture, the traditional components of a Recommender System are illustrated. In this case, the Recommender System first needs an acquisition component to collect user signals such as website interactions, newsletter clicks, purchases, or transactions. The data preprocessing component processes and prepares the incoming data to persist it in a relational database. In the next step computational algorithms are applied to the collected data. The aim of these algorithms is to create prediction models, e.g., itemitem-based or user-user-based to recommend appropriate items to a user.

Afterwards, the model is available in a prediction module to determine recommendations for a user. Unfortunately, this part of the architecture is mostly sufficient to create recommendations for an already known user. In other words, the architecture only works well for users who have purchased items in the past. To solve this problem we suggest to enhance the general architecture by including social information about users as depicted in the upper part of Fig. 3.

The upper part of the architecture targets to collect social information of users from external or even internal sources, if available. Here, the acquisition and preprocessing components mainly aim to interact with external application programming interfaces (APIs), such as Facebook, Twitter, YouTube, or LinkedIn. Since the retrieved data represents a graph, with nodes representing users and edges representing relationships among them, an appropriate data storage solution is given by a graph database. Besides the advantage to represent the data in the most natural way to discover social aspects, graph databases provide out-of-the-box implementations 


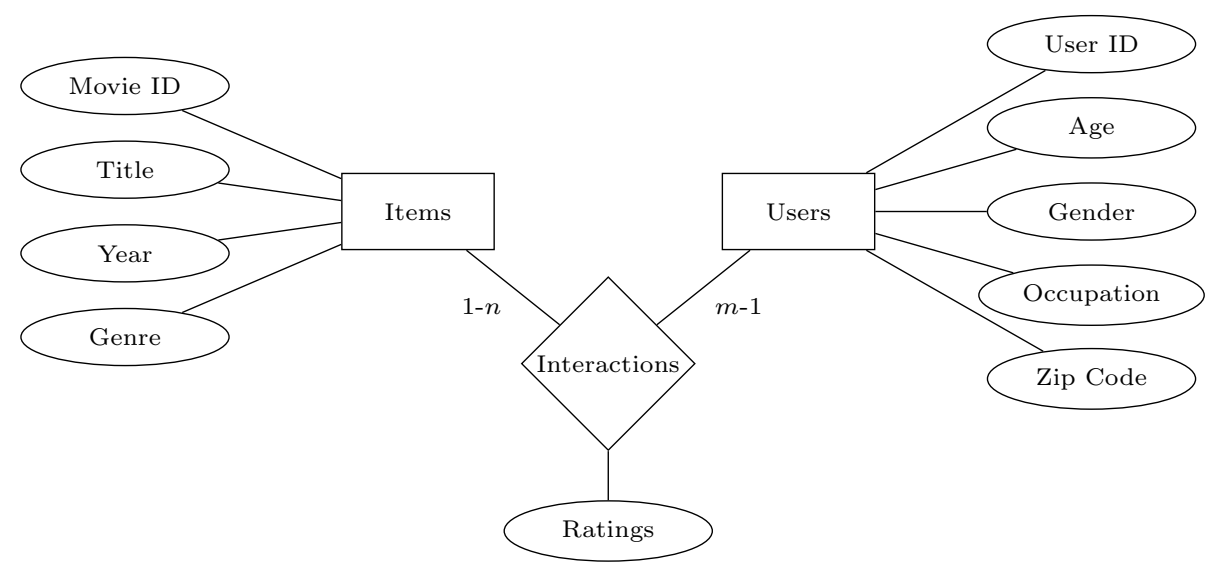

Fig. 4. Entity-Relationship Model describing our running example.

of common graph algorithms for centrality and community detection. In the following community detection step, these algorithms can be applied to determine a community model. In contrast to the collaborative filtering model, the community detection is independent of historical user information such as purchases, since it only considers the relationships among the users; however, to calculate the community model itself the historical data is needed. Therefore, the enhancement represents a natural fit to alleviate the cold-start problem. At this point, we introduce a possible implementation of the general architecture as depicted in Fig. 3.

\subsection{Data models and storage}

Our introduced general architecture is built upon two data models: a relational data model and a graph data model.

The relational data model is described in the form of a generic Entity-Relationship Model (ERM) as shown in Fig. 4. It is generic by the usage of abstract entities as Items, Users, and Interactions. The attributes used in the data model depend on the items to be recommended such as books, songs, services, or basically everything a Recommender System can be built for. For a better understanding, entity Items in Fig. 4 contains attributes for movie recommendations. On the other hand, entity Users describes all information a company collects and stores about their users such as, e.g., user id, age, gender, age, occupation, or zip code. The Interactions relationship describes the interactions of users with items. In the process, interactions represent purchases, browsing a companies' website, or indicating interest by clicking a newsletter. For illustration, relationship Interactions in the data model represents ratings of movies. As this data model is based on an ERM a relational database is suitable as a realization. In our implementation, we use a MySQL ${ }^{\mathrm{b}}$ to store items, interactions, and users.

b https://www.mysql.com. 


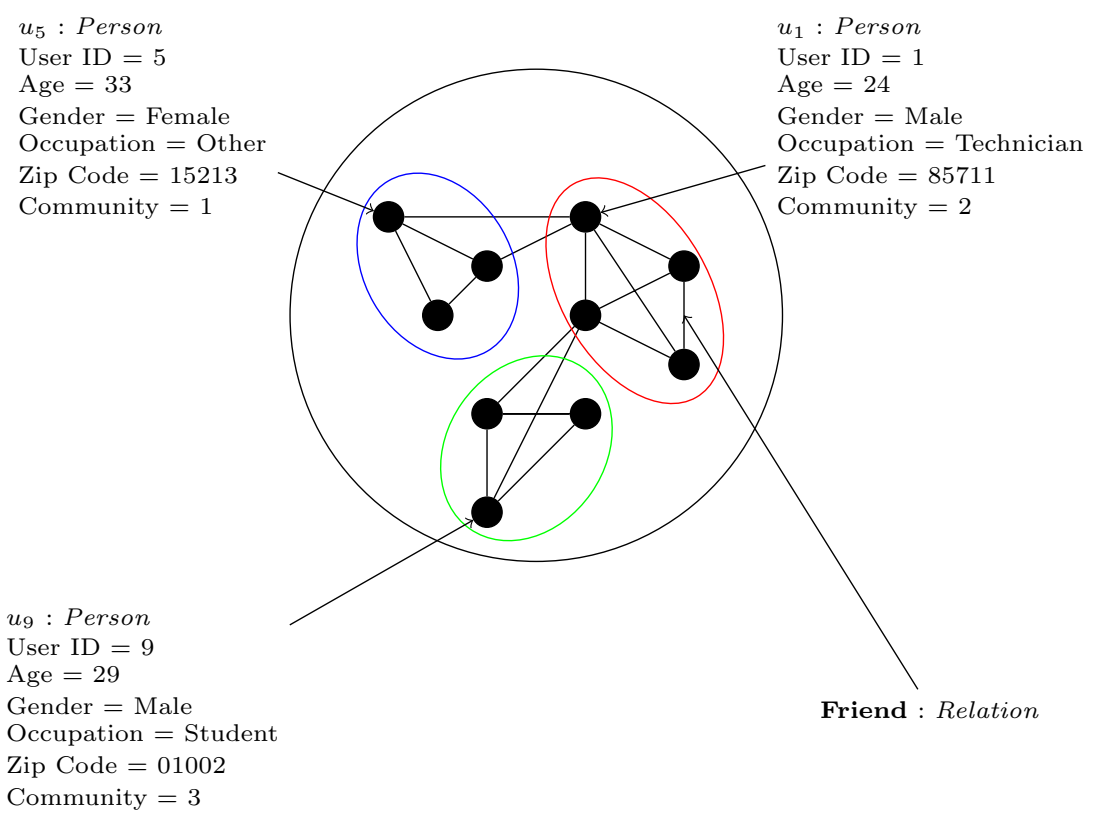

Fig. 5. (Color online) Graph Data Model. The social graph contains three communities represented by green, red, and blue ellipses. Each user of a community is characterized by her user id, age, gender, occupation, zip code, and community. The edges among the nodes represent a friendship.

The Graph Data Model is depicted in Fig. 5 and represents a social graph. As already mentioned, nodes in a social graph represent users and edges relationships among them. In Fig. 5 the edges represent friendship and the nodes represent users who are labeled as type Person. Type Person consists of attributes such as user id, age, gender, occupation, zip code, and an associated community (after the community detection was applied to the social graph). The data models of graph databases are flexible in the sense that they do not rely on a schema as a relational database does. Therefore, existing nodes can be extended and new node types included, which applies to edges as well. As we are interested in applying common graph algorithms on the social graph, we decided to use Neo4j. ${ }^{c}$ Neo4j provides algorithms ${ }^{\mathrm{d}}$ for centrality and community detection.

\subsection{Acquisition and preprocessing}

Before graph algorithms can be applied a dataset needs to be available in the graph database. In the general case, this data is provided by famous social networks such as Facebook, Twitter, YouTube, or LinkedIn. Via their official APIs user information and user relationships can be retrieved and aligned with the already collected data in a relational database. In the proposed implementation we use data provided as CSV

${ }^{\mathrm{c}}$ http://neo4j.com.

$\mathrm{d}_{\text {http://neo4j.com/developer/graph-algorithms. }}$ 
files, which contains demographic user information such as age, gender, occupation, and zip code. Thereby, the processing part is implemented in Python and takes advantage of the official Neo4j database driver ${ }^{\mathrm{e}}$ to ingest the data into a graph database. The following statement indicates how to use the driver to run a Cypher graph query ${ }^{\mathrm{f}}$ to load users and create random friendships based on an uniform distribution:

LOAD CSV FROM 'file://users.csv' AS row FIELDTERMINATOR '|' CREATE (p: Person\{id:toInteger (row [0]), age:toInteger(row [1]), gender:row [2], occupation:row [3], zipCode:toInteger (row [4])\});

MATCH (p1:Person), (p2:Person) WITH p1, p2 WHERE ( $($ and ()$<0.1)$ AND (p1. id $<>$ p2.id)) MERGE (p1)-[:friend $]-(\mathrm{p} 2)$;

Here, for each line in the CSV file, a node of type Person with an identifier, age, gender, occupation, and zip code is created in the graph database. In Sec. 4, we describe different approaches to create edges among nodes in more detail.

\subsection{Community detection}

The general idea of community detection is to identify underlying structural patterns in a given social graph. More specifically, a community describes a subset of a social graph in which the users share a common characteristic, such as being friends, colleagues, or neighbors. Multiple approaches exist to determine communities in a social graph, such as cliques or betweenness. The community detection approach we propose is based on the Louvain algorithm. ${ }^{6}$ In contrast to other community detection algorithms, the Louvain algorithm is considerably fast due to its heuristic greedy approach. The computational runtime to determine communities within a graph with $n$ nodes takes $O(n \log (n))$ in most cases, which is appropriate for detecting communities even within a large social network, however an exact proof of this computational efficiency is not given so far. We now focus on our empirical evaluation.

\section{Empirical Evaluation}

In this section, we experimentally evaluate our proposed solution. We are particularly interested in investigating the quality of recommendations produced when social-based signals are considered in the estimation of new user's ratings.

We have used the well-known and well-understood MovieLens $100 \mathrm{~K}^{\mathrm{g}}$ dataset which contains information about 100,000 ratings of 943 users for 1,682 movies. The dataset also contains user's demographic information such as age, gender, occupation, and zip code, and movie-related information such as title, genre, and release date.

${ }^{\mathrm{e}} \mathrm{https}$ //neo4j.com/developer/python.

${ }_{\mathrm{f}}$ https://neo4j.com/developer/cypher-query-language/.

$\mathrm{g}_{\text {http://files.grouplens.org/datasets/movielens/ml-100k-README.txt. }}$ 
User ratings, movie information, and demographic data about the user were stored in a MySQL database. Social network data (i.e., the social graph) was stored in a Neo4j database.

Due to the lack of appropriate datasets comprehending both social information about users and how these users have rated items - the MovieLens dataset does not contain any information about how users are socially related —, a social graph $G(V, E)$ has to be constructed, where nodes in $V$ represent the complete set of users in the dataset and edges $E$ represent social relationships among users. We build $G$ by creating an edge between two users $u_{i}$ and $u_{j}$ from $V$ such that $i \neq j$ if and only if $\tau\left(u_{i}, u_{j}\right)=1$, where $\tau\left(u_{i}, u_{j}\right)$ is a Boolean function. Equation (1) presents a simple model of $\tau\left(u_{i}, u_{j}\right)$, where $\operatorname{rel}\left(u_{i}, u_{j}\right)$ represents an edge assignment (i.e., relatedness) strategy. Note that we considered a random component rand to account for plausible relationships that are established at random. In this sense, whenever rand $<\sigma$, an edge is assigned. In our experiments, we considered several values for $\sigma$, as depicted in Table 1:

$$
\tau\left(u_{i}, u_{j}\right)= \begin{cases}1 & \text { if } \operatorname{rel}\left(u_{i}, u_{j}\right) \vee \operatorname{rand}<\sigma \\ 0 & \text { otherwise }\end{cases}
$$

Edge assignment. We defined and compared different relatedness strategies in this work. First, we randomly assign edges between nodes. Here, we refer to this strategy as $\operatorname{PR}\left(u_{i}, u_{j}\right)$, where $\mathrm{PR}$ stands to pure random. This is equivalent to define $\operatorname{rel}\left(u_{i}, u_{j}\right)=0$. Second, we defined more elaborate strategies that consider user's demographic information to assign edges between users. We highlight that each strategy can be plugged into Eq. (1) as described next.

The $\operatorname{rel}_{a}\left(u_{i}, u_{j}\right)$ strategy assumes that two users $u_{i}$ and $u_{j}$ are socially connected if they have similar ages, as depicted in Eq. (2). The $\operatorname{rel}_{a o}\left(u_{i}, u_{j}\right)$ strategy combines the

Table 1. Summary of results obtained by different combinations of demographic user attributes that were used to create the social graph. Each entry represents the average of 10 algorithm executions.

\begin{tabular}{lccccr}
\hline Method & $\sigma$ & RMSE & MAE & Communities & \multicolumn{1}{c}{ Edges } \\
\hline $\operatorname{PR}\left(u_{i}, u_{j}\right)$ & 0.100 & 1.28 & 1.09 & 8.8 & $84,362.7$ \\
$\operatorname{PR}\left(u_{i}, u_{j}\right)$ & 0.150 & 1.27 & 1.08 & 8.3 & $123,266.5$ \\
$\operatorname{PR}\left(u_{i}, u_{j}\right)$ & 0.200 & 1.25 & 1.08 & 7.2 & $159,832.4$ \\
$\operatorname{rel}_{a}\left(u_{i}, u_{j}\right)$ & 0.015 & 1.19 & 1.05 & 4.5 & $86,942.2$ \\
$\operatorname{rel}_{a}\left(u_{i}, u_{j}\right)$ & 0.070 & 1.19 & 1.05 & 4.5 & $125,769.1$ \\
$\operatorname{rel}_{a}\left(u_{i}, u_{j}\right)$ & 0.120 & 1.19 & 1.05 & 4.3 & $158,926.3$ \\
$\operatorname{rel}_{a o}\left(u_{i}, u_{j}\right)$ & 0.090 & 1.23 & 1.07 & 5.8 & $86,425.2$ \\
$\operatorname{rel}_{a o}\left(u_{i}, u_{j}\right)$ & 0.140 & 1.22 & 1.07 & 5.4 & $124,739.7$ \\
$\operatorname{rel}_{a o}\left(u_{i}, u_{j}\right)$ & 0.185 & 1.23 & 1.07 & 5.8 & $157,078.8$ \\
$\operatorname{rel}_{a o z}\left(u_{i}, u_{j}\right)$ & 0.099 & 1.26 & 1.08 & 8.3 & $83,630.1$ \\
$\operatorname{rel}_{a o z}\left(u_{i}, u_{j}\right)$ & 0.149 & 1.26 & 1.08 & 8.0 & $122,501.3$ \\
$\operatorname{rel}_{a o z}\left(u_{i}, u_{j}\right)$ & 0.199 & 1.26 & 1.08 & 7.9 & $159,086.1$ \\
\hline
\end{tabular}


previous $\operatorname{rel}_{a}\left(u_{i}, u_{j}\right)$ strategy with an assessment of users' occupation. Equation (3) describes the underlying rationale of this strategy. Finally, $\operatorname{rel}_{a o z}\left(u_{i}, u_{j}\right)$ considers the users' age, occupation, and zip code, all together:

$$
\begin{gathered}
\operatorname{rel}_{a}\left(u_{i}, u_{j}\right)= \begin{cases}1 & \text { if } \operatorname{abs}\left(\operatorname{ag}_{i}-\operatorname{ag}_{j}\right) \leq 3, \\
0 & \text { otherwise, }\end{cases} \\
\operatorname{rel}_{a}\left(u_{i}, u_{j}\right)= \begin{cases}1 & \text { if } \operatorname{rel}_{a}\left(u_{i}, u_{j}\right) \wedge \mathrm{oc}_{i}=\mathrm{oc}_{j}, \\
0 & \text { otherwise, }\end{cases} \\
\operatorname{rel}_{a o z}\left(u_{i}, u_{j}\right)= \begin{cases}1 & \text { if rel } \operatorname{rel}_{a o}\left(u_{i}, u_{j}\right) \wedge \mathrm{zc}_{i}=\mathrm{zc}_{j}, \\
0 & \text { otherwise, }\end{cases}
\end{gathered}
$$

where $\mathrm{ag}_{n}, \mathrm{Oc}_{n}$, and $\mathrm{zc}_{n}$ are, respectively, the age, the occupation, and the zip code of user $n \in i, j$.

Metrics. Two statistical metrics were selected to measure the correctness of the estimated ratings, i.e., the root-mean-square error (RMSE) and the mean absolute error (MAE). RMSE and MAE are presented in Eqs. (5) and (6), respectively, and explained as follows. Given a set $\hat{Y}=\left\{\hat{y}_{1}, \hat{y}_{2}, \ldots, \hat{y}_{n}\right\}$ of estimated values and a set $Y=\left\{y_{1}, y_{2}, \ldots, y_{n}\right\}$ of observed values, the RMSE measures the deviation of estimated values and the MAE measures the average magnitude of the estimation errors.

Rating prediction. In order to calculate RMSE and MAE, we employ the leaveone-out approach, i.e., for each user $u_{i}$ in the dataset, we find a community $C$ such that $u_{i} \in C$ and calculate the aggregated ratings of each item $i_{l}$ provided by all users within $C^{\prime}=C-\left\{u_{i}\right\}$. In this work, aggregated ratings are computed using Eq. (7), where $R_{l}^{+}$ is the set of positive ratings provided by users in $C^{\prime}$ to item $i_{l}$ such that $\forall r \in R_{l}^{+}, r>0$. Then, we select the $k$ highest-rated items of $u_{i}$ and compare the original ratings for such items with the computed aggregated ratings. In our experiments, we set $k=10$. Given these two sets of ratings, we calculate the aforementioned metrics. Figure 6 exemplifies this process referring to the example provided in Sec. 3.

$$
\begin{gathered}
\operatorname{RMSE}=\sqrt{\frac{\sum_{i=1}^{n}\left(\hat{y}_{i}-y_{i}\right)^{2}}{n},} \\
\operatorname{MAE}=\frac{\sum_{i=1}^{n}\left|\hat{y}_{i}-y_{i}\right|}{n}, \\
\operatorname{agg}\left(i_{l}\right)=\frac{\sum_{r_{j} \in R_{l}^{+}}\left(r_{j}\right)}{\left|R_{l}^{+}\right|} .
\end{gathered}
$$

Providing recommendations. As our results in Table 1 show, RMSE and MAE for rated items of an unknown user by its community are both very small. To achieve a suitable comparison among the different approaches, we created social graphs with a similar amount of edges. For instance, a purely random created graph with $\sigma=0.1$ contains about 84,000 edges and leads to an RSME of 1.28. A graph considering the demographic information of the age among users additionally needs a randomness of $\sigma=0.015$ to have a similar amount of edges. As the results show, the 


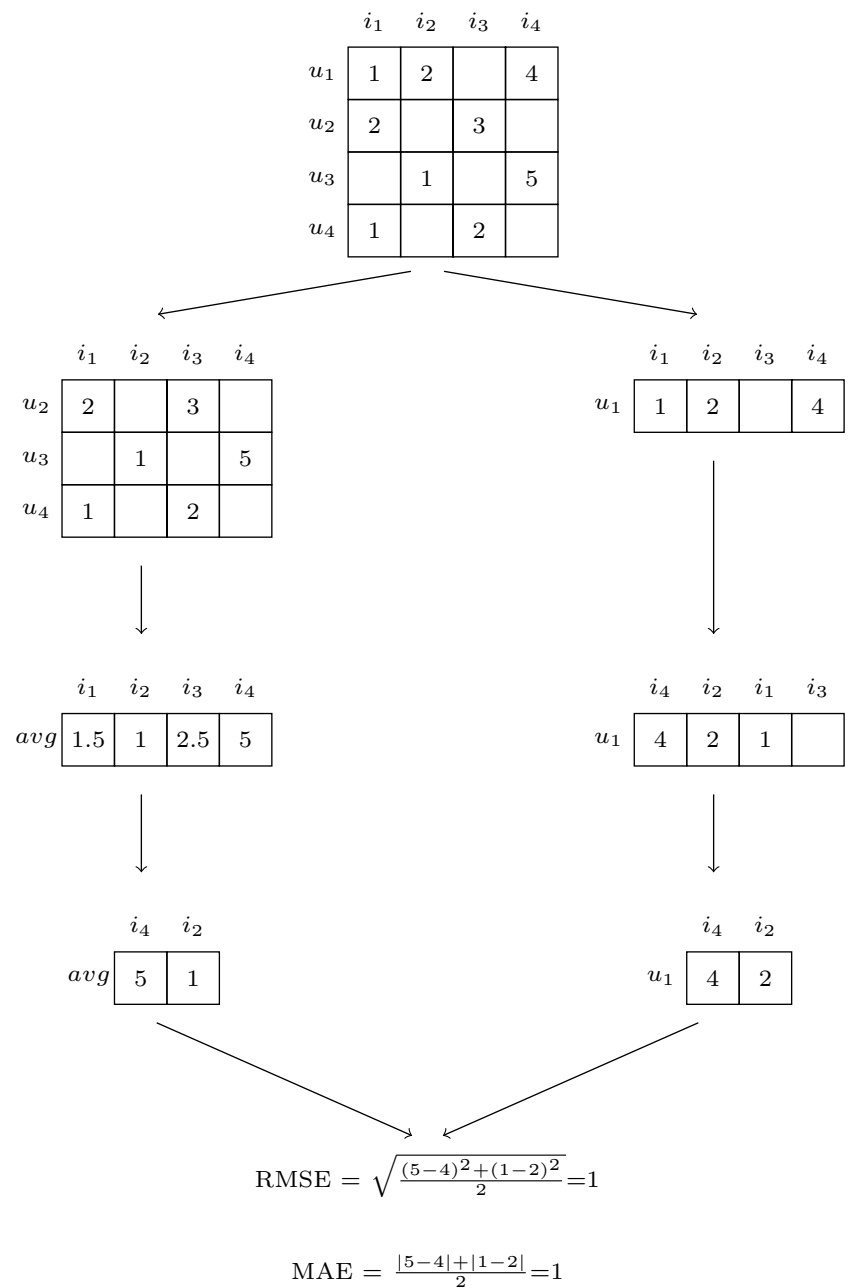

Fig. 6. Example of the rating prediction process based on Community 2 illustrated in Fig. 5. On the first level, the user-item matrix of a community consisting of $u_{1}, u_{2}, u_{3}$, and $u_{4}$ is given. Since we want to calculate the RMSE and MAE of user $u_{1}$, we split the user-item matrix into two disjoint parts. The left part contains ratings of all community members except for user $u_{1}$. The right part contains only the ratings given by user $u_{1}$. In the next step, we calculate the aggregated ratings given by all remaining users (i.e., left part) to items $i_{1}, i_{2}, i_{3}$, and $i_{4}$. Finally, to calculate the RMSE and MAE, we considered only the top $k=2$ highest-rated items of user $u_{1}$ and compared their ratings with $u_{1}$ 's original ratings.

RMSE for this case is better (i.e., 1.19). Generally, our approach shows an improvement of both applied metrics, except for the case in which we considered age, occupation, and zip code of users to build the social graph. To conclude, the community seems to share very similar taste about the rated items of the unknown user. Therefore, it seems to be an appropriate solution for the cold-start problem to rely on the taste of the community and recommend, for instance, the top 10 items based on the aggregated ratings of the community. 


\section{Discussion and Limitations}

The limitations of this work are twofold. First, given that no public dataset including user ratings along social and demographic information was available, we only had the option to conduct experiments using synthetic data. The produced dataset, although appropriate for the purpose of this work, does not capture the real social relationships among users. We argue that these relationships provide additional insights that may contribute to more effective recommendations. Since our synthetic social graph is only based on simple, yet plausible, user's demographic information such as age, occupation, and zip code, our obtained results should be observed with caution and be considered as a possible approach to evaluating the impact of real social data in the future.

The second limitation of this work consists of the use of only one aggregation strategy (i.e., an average-based approach to creating an overall preference estimation of users in a social community). Different aggregation approaches have to be designed and a more comprehensive analysis of their performance is planned as a future extension of this work.

\section{Related Work}

Several research efforts have investigated the problem of enhancing traditional Collaborative Filtering with additional signals derived from diverse sources. We summarize some of these relevant studies next.

Social signals. Social recommender systems were introduced by $\mathrm{Ma}$ et al. ${ }^{7}$ and different social signals have been considered since then. In the work of Wang et al. ${ }^{8}$ weak and strong ties among users were taken into account to favor recommendation. Accordingly, due to the intuition that social influence is bounded to how users trust each other's opinion, several approaches have considered social trust as a factor to modulate rating prediction. ${ }^{2}$

Curation signals. Recent studies have shown that curation can improve existing recommendation algorithms. Saaya et al. ${ }^{9}$ employed content-based recommendation algorithms for helping users to curate their multimedia content, in the context of the Scoop.it website. In their work, Latent Semantic Indexing (LSI) and Latent Dirichlet Allocation (LDA) were applied to extract content-based features, while the content itself was treated independently from its form (i.e., web page, image, video, etc.). The authors argue that the use of curated signals helps to provide better recommendation results in terms of precision metric. Similarly, Liu et al. ${ }^{10}$ enhanced a neighborhoodbased collaborative filtering model with human curation signals extracted from the Pinterest website. The rationale behind their work was to exploit the relationship among items in user's curated collections. They assume that items grouped in the same collection tend to be more similar than items in different collections. Results showed that the addition of curation-based signal outperforms classical neighborhood recommendation approaches. From another perspective, Merritt et al. ${ }^{11}$ 
developed Kurator, an intelligent, human-in-the-loop system for curating personal content. Kurator uses a three-class rating system in which users can classify their content. The hybrid approach consists of two steps: First, a machine learning algorithm (i.e., logistic regression) is applied for predicting the class of an item and filtering it based on the predicted class; and, finally, a collective intelligence approach is used, which allows a collection of users to provide subjective assessment on the filtered content.

\section{Conclusion and Future Work}

Despite its popularity and intuitive rationale, traditional Collaborative Filtering methods suffer from the so-called cold-start problem. This problem occurs when new users or items enter the system and for those no previous preference information is available. In this paper, we have demonstrated how social network data can be employed to enhance $\mathrm{CF}$ models so that the cold-start problem can be alleviated. We have presented a concrete implementation of our social-based recommendation framework and reported experimental results that highlight the usefulness of this framework. Specifically, we have demonstrated that social signals can effectively provide alternatives to produce recommendations to users with no previous rating data.

As future work, we plan to implement our approach using different graph database systems, since the provided community detection algorithms are different among those systems. This will help us to examine the impact of community detection in the overall recommendation performance with regards to performance and accuracy.

We also plan to investigate intra- and inter-community influence in produced recommendations. Our intuition is that considering the strength of social ties among the users in a community can provide useful insights on preference similarity. For instance, the strength of social ties could be used to weight user-user similarity.

\section{Acknowledgments}

This research has been financially supported by the Brazilian National Council for Scientific and Technological Development $(\mathrm{CNPq})-$ Science without Borders Program.

\section{References}

1. G. Adomavicius and A. Tuzhilin, Toward the next generation of recommender systems: A survey of the state-of-the-art and possible extensions, IEEE Trans. Knowl. Data Eng. 17, (2005) 734-749.

2. X. Yang, Y. Guo, Y. Liu and H. Steck, A survey of collaborative filtering based social recommender systems, Comput. Commun. 41 (2014) 1-16.

3. O. Oechslein and T. Hess, The value of a recommendation: The role of social ties in social recommender systems, in Proc. 201447 th Hawaii Int. Conf. System Sciences (2014), pp. 1864-1873. 
4. L. Homann, B. Maleszka, D. M. L. Martins and G. Vossen, A generic framework for collaborative filtering based on social collective recommendation, Proc. Int. Conf. Computational Collective Intelligence, eds. N. Nguyen, E. Pimenidis, Z. Khan and B. Trawiński, Lectuire Notes in Computer Science, Vol. 11055 (Springer, Cham, 2018), pp. 238-247.

5. J. Leskovec, A. Rajaraman and J. D. Ullman, Mining of Massive Datasets (Cambridge University Press, 2014).

6. V. D. Blondel, J. L. Guillaume, R. Lambiotte and E. Lefebvre, Fast unfolding of communities in large networks, J. Stat. Mech. Theory Exp. 2008, (2008) P10008.

7. H. Ma, H. Yang, M. R. Lyu and I. King, SoRec: Social recommendation using probabilistic matrix factorization, in Proc. 17th ACM Conf. Information and Knowledge Management (ACM, 2008), pp. 931-940.

8. X. Wang, S. C. Hoi, M. Ester, J. Bu and C. Chen, Learning personalized preference of strong and weak ties for social recommendation, in Proc. 26th Int. Conf. World Wide Web (International World Wide Web Conferences Steering Committee, 2017), pp. 1601-1610.

9. Z. Saaya, R. Rafter, M. Schaal and B. Smyth, The curated web: A recommendation challenge, in Proc. 7th ACM Conf. Recommender Systems (ACM Press, New York, 2013), pp. 101-104.

10. Y. Liu, D. Chechik and J. Cho, Power of human curation in recommendation system, in Proc. 25th Int. Conf. Companion on World Wide Web (2016), pp. 79-80.

11. D. Merritt, J. Jones, M. S. Ackerman and W. S. Lasecki, Kurator: Using the crowd to help families with personal curation tasks, in Proc. 2017 CSCW Conf. Computer Supported Cooperative Work (ACM Press, New York, 2017). 